\title{
Security Cost Analysis with Linear Ramp Model using Contingency Constrained Optimal Power Flow
}

\author{
Jae-kun Lyu*, Mun-Kyeom Kim ${ }^{\dagger}$ and Jong-Keun Park*
}

\begin{abstract}
This paper proposes a novel technique for calculating the security costs that properly includes ramping constraints in the operation of a deregulated power system. The ramping process is modeled by a piecewise linear function with certain assumptions. During this process, a ramping cost is incurred if the permissible limits are exceeded. The optimal production costs of the power producers are calculated with the ramping cost included, considering a time horizon with $\mathrm{N}-1$ contingency cases using contingency constrained optimal power flow (CCOPF), which is solved by the primal-dual interior point method (PDIPM). A contingency analysis is also performed taking into account the severity index of transmission line outages and its sensitivity analysis. The results from an illustrative case study based on the IEEE 30-bus system are analyzed. One attractive feature of the proposed approach is that an optimal solution is more realistic than the conventional approach because it satisfies physical constraints, such as the ramping constraint.
\end{abstract}

Keywords: security cost, piecewise linear ramping model, optimal power flow, interior point method

\section{Introduction}

The modern electric power business is rapidly becoming market driven. In the deregulated power industry, end users can actively participate in the market to purchase power with whomever they choose, while suppliers try to make more profit by competing with each other. Although the promise of economic benefits is driving deregulation, one cannot lose sight of security requirements while implementing this market-driven approach. Maintaining security remains the most critical aspect of power systems operation because of the increasingly important role that electric energy plays in the national economy [1]. In this regard, security cost analysis is important since it provides information to lead the operating of network to a more secure condition and also can help market participants make profitable market decisions in electricity markets.

In general, the term security cost is usually used to represent how much a company spends to maintain system security. In recent years, there has been a great deal of research about security costs from the point of view of the correlation between market operations and power system operations. In [2], security cost is defined as the amount of money spent on security improvement. This cost usually increases rapidly in proportion to increasing contingency probability and causes a rapid increase in locational marginal pricing (LMP). In [3]-[4], security costs, defined as the cost of security enhancements for contingency events, are evaluated using the value of lost load during network contingencies.

$\dagger \quad$ Corresponding Author: Dept. of Electrical Engineering, Seoul National University, Seoul, 151-742, Korea (mith33@snu.ac.kr)

* Dept. of Electrical Engineering, Seoul National University, Seoul, 151-742, Korea (handyjack@snu.ac.kr, jkpark@snu.ac.kr)

Received 11 May 2009; Accepted 7 August 2009
In real power system operations, to avoid shortening the lifetime of the turbine and preventing brittle failure, the system operator (SO) tries to keep the generator operating within safe limits. This ramping constraint should be considered in real dispatch problem to ensure power system security. With increasing interest about ramping constraints in the deregulated power market because of their relation to reserve trading, several studies that have been published recently considered ramp rate in a variety of problems. In [5], the authors suggest the effects of ramp rate limits on unit commitment and the economic dispatch problem. The Lagrangian relaxation-based method is proposed to show the short-term resource scheduling with ramp rate constraints [6]. In [7], the authors present a dispatch analysis focusing on the primary contradiction between the generation supply and load demand due to the ramping constraint of generator and regulation capacity.

There are two types of control mode that could eliminate or alleviate the threat of contingencies to system security: the preventive method and the corrective method. These two methods for managing contingencies represent different trade-offs between security and economics. So far, most optimization problems can be considered preventive since they impose additional constraints in order to allow for a feasible state in the event of contingency, but they do not take into account the system's corrective capacities after a contingency occurs. An approach that allows the controls to be reset after the occurrence of a contingency is known as a corrective method. Nowadays, contingency constrained optimal power flow (CCOPF) with postcontingency corrective rescheduling [8] has become a standard tool in the power industry because it provides the same security level as the preventive mode with a lower operating cost. However, in computing security costs the application of CCOPF with respect to the ramping con- 
straint has not yet been considered in the literature.

In this study we propose a new method for calculating the security cost that properly includes ramping constraints in the operation of a deregulated power system. We define the security cost as the additional cost to ensure system stability, considering line outages and different kinds of physical constraints, including the ramp limit. Here, additional means the difference of production cost between the base-case solution and the contingency-case solution in CCOPF. The optimal production costs are computed with ramping cost, considering piecewise linear ramp rate limits using CCOPF with corrective rescheduling. During this ramping process, the ramping cost is invoked when operators violate system operational limits. The CCOPF problem for security cost analysis can be solved by the primal-dual interior-point method (PDIPM), exploiting the structure of the problem.

The rest of this paper is organized as follows. Section 2 describes the modeling of a ramping process. Section 3 presents the formulation of the problem with PDIPM, and the contingency analysis is presented in Section 4. Section 5 presents the result for the IEEE IEEE-30 bus system obtained using both a conventional OPF and the one proposed in this paper. Finally, Section 6 summarizes the conclusions drawn from the study.

\section{Ramping Process}

There are some assumptions that can be used to simplify the application of the ramping process for our proposed approach. The ramping process can be thought as a function of time, and it can be simplified as a combination of a few linear ramping functions in which the ramp rates are fixed within a certain time period. When a generating unit is ramping up or down from one moment to the next, it can remain at the original loading level for a while, is referred to as ramping delay. For the sake of simplicity, we do not consider ramping delays, and a piecewise linear ramping function is assumed. According to the unit's condition, the ramp limit can be determined as follows: normal operating conditions, start-up, and shutdown. Only the normal operating ramp rate is considered since we are interested in the dispatch problem, not the unit commitment (UC) problem. Start-up and shutdown ramp rates are relevant to the UC problem, which is an hourly scheduling problem. Accordingly, we make the following assumptions in this article:

1) Ramping delay is not considered.

2) Piecewise linear ramping function is accepted.

3) Only normal operating ramp rate is considered.

In this picture, the ramping process with a piecewise linear ramping function can be modeled as shown in Fig 1.

where $\alpha_{m}$ is the linear ramp rate which differs from segment to segment. The power output of unit $i$ for the first period, $\left(0, \mathrm{~T}_{1}\right)$, is given by following equation [9].

$$
P_{i}=\alpha_{1} \cdot R T+P_{i 1}
$$

where RT is the total ramping time of unit $i$. Similarly, the power output of unit $i$ for the second period, $\left(T_{1}, T_{2}\right)$, is given by

$$
P_{i}=\alpha_{2} \cdot\left(R T-T_{1}\right)+P_{i 2}
$$

With $m$ segments, the power output of unit $i$ in any time during the piecewise linear ramping period $\left(0, \mathrm{~T}_{\mathrm{m}}\right)$ is generally described as follows;

$$
P_{i}=\alpha_{m} \cdot\left(R T-T_{m}\right)+P_{i m}, 0<R T<T_{m}
$$

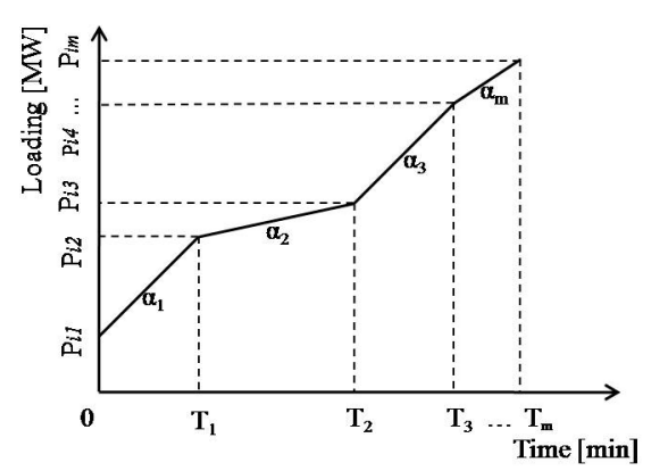

Fig. 1. Piecewise linear ramping process

\section{Problem Formulation}

\subsection{Overview of CCOPF with ramping constraint}

The objective function for evaluating the security cost is a multi-period optimization problem that minimizes the production cost for producing electricity. The objective function of the CCOPF problem with a ramping constraint can be expressed by equation (4).

$$
\min \sum_{i=1}^{n} \sum_{t=1}^{n t}\left(C_{i}\left(P_{i t}\right)+R C_{i t}\right)
$$

where $C_{i}\left(P_{i t}\right)$ and $R C_{i t}$ are the fuel cost and ramping cost, respectively, of the $i$ th generator at time $t$. The fuel cost function is described by a quadratic function with fuel cost coefficients. When generators operate within their ramp limit, the ramping cost $R C_{i t}$ will be zero. If they violate their ramp limit, it may shorten the lifetime of generator rotor. To avoid this, system operators try to keep the generator operating within safe limits. Then additional production cost beyond the safe limit is charged as a ramping cost. The objective function should satisfy both equality and inequality constraints. The equality constraint is the system real power balance, and the inequalities include unit generation limits, the power limit on the transmission line, the ramp limit, and system voltage limits.

\section{System real power balance:}

$$
\begin{aligned}
& \quad P_{D t}-\sum_{\mathrm{all} i} P_{i t}=P_{L t} \quad t=1,2, \ldots, n t \\
& \text { Power output limits: } \\
& P_{i, \text { min }} \leq P_{i t} \leq P_{i, \text { max }} \quad i=1,2, \ldots N \quad \text { and } t=1,2, \ldots, n t
\end{aligned}
$$

Transmission flow limits from bus $k$ to bus $m$ :

$$
P_{k m, \min } \leq P_{k m t} \leq P_{k m, \max } \quad i=1,2, \ldots N
$$


System voltage limits:

Ramp rate limit:

$$
V_{\min } \leq V_{t} \leq V_{\max }
$$

$P_{i t-1}-r_{i d} \leq P_{i t} \leq P_{i t-1}+r_{i u} \quad i=1,2, N$ and $t=1,2, \ldots, n t$

where $r_{i d}$ and $r_{i u}$ are, respectively, the lower and upper ramp rate limits on the generation level of unit $i$ during period $t$.

\subsection{Solution approach}

The interior point method (IPM) is getting more attention from power system engineers because of its ability to solve problems faster and more efficiently than with LP methods. In this paper, we employ the primal-dual interior point method (PDIPM). This method is Similar to established methods in the literature [10]-[12]. Since the primaldual variant is the most theoretically elegant of the many variants and also the most successful computationally, the PDIPM is a powerful tool for solving the security cost problem. Our proposed formulation can be written mathematically as:

subject to:

$$
\begin{gathered}
\min f\left(\boldsymbol{x}_{\boldsymbol{t}}\right) \\
g\left(\boldsymbol{x}_{\boldsymbol{t}}\right)=0 \\
h_{\text {min }} \leq h\left(\boldsymbol{x}_{\boldsymbol{t}}\right) \leq h_{\max } \\
r d \leq r\left(\boldsymbol{x}_{\boldsymbol{t}}\right) \leq r u
\end{gathered}
$$

where $x_{t} \in \mathrm{R}^{\mathrm{m}}$ is the vector of optimization variables for time $t$ and $r\left(x_{t}\right)=P_{i t}-P_{i t-1}$. The optimization variables for each time interval are arranged in blocks as $\boldsymbol{x}_{\boldsymbol{t}}=\left[\boldsymbol{P}^{\mathrm{T}}{ }_{i t}, \boldsymbol{Q}^{\mathrm{T}}{ }_{i t}\right.$, $\left.\boldsymbol{V}_{t}^{\mathrm{T}}\right]^{\mathrm{T}}(t=1,2, \ldots, n t)$, including the real power generation and the bus voltages. The overall variables are arranged and expressed as $\boldsymbol{x}=\left[\boldsymbol{x}_{1}^{\mathrm{T}}, \boldsymbol{x}^{\mathrm{T}}{ }_{2}, \ldots, \boldsymbol{x}_{n t}^{\mathrm{T}}\right]^{\mathrm{T}}(t=1,2, \ldots n t)$. The ramping function $r\left(\boldsymbol{x}_{\boldsymbol{t}}\right)$ would be a time interval constraint because of its essential property, so it is different to take the ramp limit into consideration in this dispatch problem so far. Here let us assume that the generation level at time $t-1$ is given. Then it can be thought of as a function of $x_{t}$ because $P_{i t-l}$ is just a constant value. In this way we can express the proposed method by PDIPM. To obtain the security cost (SC), the production cost (PC) over a given time period should be computed by the proposed method in both base and contingency cases. To implement the algorithm, we introduce the slack variable vectors (primal variable vectors) $(l, u, d, p) \geq 0$. As a result of the inequality constraints, Equation (10) can be converted to:

$$
\left\{\begin{array}{c}
h\left(x_{t}\right)-h_{\min }-l=0 \\
h\left(x_{t}\right)-h_{\max }+u=0 \\
r\left(x_{t}\right)-r d-d=0 \\
r\left(x_{t}\right)-r u+p=0
\end{array}\right.
$$

After adding a logarithmic barrier function, the Lagrangian for the problem is constructed as the following equation (12):

$$
\begin{array}{r}
L\left(x_{t}, y, z, w, s, t, l, u, d, p\right)=f\left(x_{t}\right)-y^{\mathrm{T}} g\left(x_{t}\right)- \\
z^{\mathrm{T}}\left(h\left(x_{t}\right)-l-h_{\min }\right)-w^{\mathrm{T}}\left(h\left(x_{t}\right)+u-h_{\max }\right)- \\
s^{\mathrm{T}}\left(r\left(x_{t}\right)-d-r d\right)-t^{\mathrm{T}}\left(r\left(x_{t}\right)+p-r u\right)- \\
\mu\left(\sum \ln u_{i}+\sum \ln l_{i}+\sum \ln d_{i}+\sum \ln p_{i}\right)
\end{array}
$$

Then, the KKT equations for the system can be derived as follows:

$$
\begin{aligned}
& \left\{\begin{array}{l}
L_{x_{t}}=\nabla f\left(x_{t}\right)-\nabla g\left(x_{t}\right) y-\nabla h\left(x_{t}\right)(z+w)=0 \\
L_{y}=g\left(x_{t}\right)=0 \\
L_{z}=h\left(x_{t}\right)-l-h_{\min }=0 \\
L_{w}=h\left(x_{t}\right)+u-h_{\max }=0 \\
L_{s}=r\left(x_{t}\right)-d-r d=0 \\
L_{t}=r\left(x_{t}\right)+p-r u=0
\end{array}\right. \\
& \left\{\begin{array}{l}
L_{l}^{\mu}=[l][z] e-\mu e=0 \\
L_{u}^{\mu}=[u][w] e+\mu e=0 \\
L_{d}^{\mu}=[d][s] e-\mu e=0 \\
L_{p}^{\mu}=[p][t] e+\mu e=0
\end{array}\right.
\end{aligned}
$$

The equations (14) are complimentary conditions, which are nonlinear. To overcome this difficulty, we introduced the perturbed factor, which decreases to zero as the algorithm is iterated many times. Thus $L_{l}^{\mu}, L_{u}^{\mu}, L_{d}^{\mu}, L_{p}^{\mu}$ are perturbed complementary conditions, and, by applying Newton's method, we can obtain the correction equations.

$$
\begin{aligned}
& \left\{\begin{array}{l}
L_{x_{t} 0}=\left(\nabla^{2} g\left(x_{t}\right) y-\nabla^{2} h\left(x_{t}\right)(z+w) \Delta x_{t}-\right. \\
\nabla^{2} r\left(x_{t}\right)(s+t)-\nabla^{2} f\left(x_{t}\right) \Delta x_{t}+g\left(x_{t}\right) \Delta y \\
+\nabla h\left(x_{t}\right)(\Delta z+\Delta w)+\nabla r\left(x_{t}\right)(\Delta s+\Delta t) \\
-L_{y 0}=\nabla g\left(x_{t}\right)^{\mathrm{T}} \Delta x_{t} \\
-L_{z 0}=\nabla h\left(x_{t}\right)^{\mathrm{T}} \Delta x_{t}-\Delta l \\
-L_{w 0}=\nabla h\left(x_{t}\right)^{\mathrm{T}} \Delta x_{t}+\Delta u \\
-L_{s 0}=r\left(x_{t}\right)^{\mathrm{T}} \Delta x_{t}-\Delta d \\
-L_{t 0}=r\left(x_{t}\right)^{\mathrm{T}}+\Delta p
\end{array}\right. \\
& \left\{\begin{array}{l}
-L_{l 0}^{\mu}=L \Delta z+Z \Delta l \\
-L_{u 0}^{\mu}=U \Delta w+W \Delta u \\
-L_{d 0}^{\mu}=D \Delta s+S \Delta d \\
-L_{p 0}^{\mu}=P \Delta t+T \Delta p
\end{array}\right.
\end{aligned}
$$

where $\left(L_{x_{t} 0}, L_{y 0}, L_{z 0}, L_{w 0}, L_{s 0}, L_{t 0}, L_{l 0}^{\mu}, L_{u 0}^{\mu}, L_{d 0}^{\mu}, L_{p 0}^{\mu}\right)$ denote the residuals of the perturbed KKT equations. $\nabla^{2} g\left(x_{t}\right), \nabla^{2} h\left(x_{t}\right)$ and $\nabla^{2} r\left(x_{t}\right)$ are Hessian matrices of $g\left(x_{t}\right), h\left(x_{t}\right)$ and $r\left(x_{t}\right)$, respectively.

$$
\begin{aligned}
& \left\{\begin{array}{c}
\Delta l=\nabla h\left(x_{t}\right)^{\mathrm{T}} \Delta x_{t}+L_{z 0} \\
\Delta u=-\left(\nabla h\left(x_{t}\right)^{\mathrm{T}} \Delta x_{t}+L_{w 0}\right) \\
\Delta d=\nabla r\left(x_{t}\right)^{\mathrm{T}} \Delta x_{t}+L_{s 0} \\
\Delta p=-\left(\nabla r\left(x_{t}\right)^{\mathrm{T}} \Delta x_{t}+L_{t 0}\right)
\end{array}\right. \\
& \left\{\begin{array}{c}
\Delta z=-L^{-1} Z \nabla h\left(x_{t}\right)^{\mathrm{T}} \Delta x-L^{-1}\left(\mathrm{Z} L_{z 0}+L_{l 0}^{\mu}\right) \\
\Delta w=U^{-1} W \nabla h\left(x_{t}\right)^{\mathrm{T}} \Delta x+U^{-1}\left(W L_{w 0}-L_{u 0}^{\mu}\right) \\
\Delta s=-D^{-1} S \nabla r\left(x_{t}\right)^{\mathrm{T}} \Delta x-D^{-1}\left(S L_{s 0}+L_{d 0}^{\mu}\right) \\
\Delta t=P^{-1} \operatorname{Tr}\left(x_{t}\right)^{\mathrm{T}} \Delta x+P^{-1}\left(T L_{t 0}-L_{p 0}^{\mu}\right)
\end{array}\right.
\end{aligned}
$$

By substituting Equation (18) into the $L_{x_{t} 0}$ term of Equation (15), the reduced correction Equation (19) is obtained at the following of linear equations:

$$
\left[\begin{array}{ccc}
H(\cdot) & \vdots & J\left(x_{t}\right)^{\mathrm{T}} \\
\cdots \cdots \cdots & \cdots & \cdots \cdots \\
J\left(x_{t}\right) & \vdots & 0
\end{array}\right]\left[\begin{array}{c}
\Delta x_{t} \\
\cdots \\
\Delta y
\end{array}\right]=-\left[\begin{array}{c}
\psi(\cdot, \mu) \\
\cdots \cdots \cdots \\
g\left(x_{t}\right)
\end{array}\right]
$$




$$
\left\{\begin{array}{l}
H(\cdot) \equiv \\
\quad\left(\nabla^{2} g\left(x_{t}\right) y-\nabla^{2} h\left(x_{t}\right)(z+w)-\nabla^{2} r\left(x_{t}\right)(s+t)-\nabla^{2} f\left(x_{t}\right)\right) \\
\quad+\nabla g\left(x_{t}\right) A \nabla g\left(x_{t}\right)^{\mathrm{T}}+\nabla r\left(x_{t}\right) B \nabla r\left(x_{t}\right)^{\mathrm{T}} \\
\quad \text { here, } A \equiv U^{-1} W-L^{-1} Z, B \equiv P^{-1} T-D^{-1} S \\
J\left(x_{t}\right) \equiv \nabla g\left(x_{t}\right)^{\mathrm{T}} \\
\psi(\cdot, \mu) \equiv-L_{x_{t} 0}+\nabla h\left(x_{t}\right)\left(U^{-1}\left(W L_{w 0}-L_{u 0}^{\mu}\right)-L^{-1}\left(\mathrm{Z} L_{z 0}+L_{l 0}^{\mu}\right)\right) \\
\quad+\nabla r\left(x_{t}\right)\left(P^{-1}\left(T L_{t 0}-L_{p 0}^{\mu}\right)-D^{-1}\left(S L_{s 0}+L_{d 0}^{\mu}\right)\right)
\end{array}\right.
$$

According to quations (10)-(20), we can safely say that the ramping constraint is included in the reduced correction equation and thought of as just like an inequality constraint. In other words, if we know the operating point at time $t-1$, then a time interval constraint, such as the ramping constraint, can be solved by the proposed method.

Next, we determine the step size and the perturbed factor for the iterative calculation. The step size is determined based on the non-negative conditions of the slack variables $(l, u, d, p) \geq 0$, which should preserve the feasibility of all the problem variables. The PDIPM uses two different step sizes for primal and dual variables. This scheme is generally more efficient than the unified single step size for all variables. Since we consider inter-temporal optimization problems in this paper, we need to introduce different step sizes for appropriate convergence characteristics for each time period. Finally, the step sizes are determined using inter-temporal slack variables and Lagrangian multipliers. To prevent non-negative variables from being zero, a small constant is multiplied to step size; in this article, the value of 0.9995 is used.

$\alpha_{P T}=0.9995 \min \left\{\frac{-l_{T}}{\Delta l_{T}}: \Delta l_{T}<0 ; \frac{-u_{T}}{\Delta u_{T}}: \Delta u_{T}<0 ; \frac{-d_{T}}{\Delta d_{T}}: \Delta d_{T}<0 ; \frac{-p_{T}}{\Delta p_{T}}: \Delta p_{T}<0 ; 1\right\}$

$\alpha_{D T}=0.9995 \min \left\{\frac{-z_{T}}{\Delta z_{T}}: \Delta z_{T}<0 ; \frac{-w_{T}}{\Delta w_{T}}: \Delta w_{T}>0 ; \frac{-s_{T}}{\Delta s_{T}}: \Delta s_{T}<0 ; \frac{-t_{T}}{\Delta t_{T}}: \Delta t_{T}>0 ; 1\right\}$

The iterative computation to find an optimal solution is performed:

$$
\left[\begin{array}{l}
x \\
l \\
u \\
d \\
p
\end{array}\right]^{k+1}=\left[\begin{array}{l}
x \\
l \\
u \\
d \\
p
\end{array}\right]^{k}+\alpha_{P}\left[\begin{array}{c}
\Delta x \\
\Delta l \\
\Delta u \\
\Delta d \\
\Delta p
\end{array}\right],\left[\begin{array}{c}
y \\
z \\
w \\
s \\
t
\end{array}\right]^{k+1}=\left[\begin{array}{c}
y \\
z \\
w \\
s \\
t
\end{array}\right]^{k}+\alpha_{D}\left[\begin{array}{c}
\Delta y \\
\Delta z \\
\Delta w \\
\Delta s \\
\Delta t
\end{array}\right]
$$

The determination of a reasonable perturbed factor is a crucial process for determining the convergence of PDIPM. Similar to the choice of step size, the perturbed factor $\mu$ for each time period is considered and determined by the following:

$$
\mu=\frac{-l^{\mathrm{T}} z+u^{\mathrm{T}} w-d^{\mathrm{T}} s+p^{\mathrm{T}} t}{2 n \beta}
$$

where $n$ is the number of variables in this problem and $\beta$ is a damping factor $(0<\beta<1)$.

\section{Contingency Analysis}

There are various factors that may affect the state of power system security, such as line outages, which may occur without warning. To deal with contingency screening, the critical contingencies were selected based on the relative values of the real power performance index (PI) given in [13], which measures the deviation from rated values of system variables, such as a branch flow or voltage. In this work, we focus only on transmission line outages for contingency analysis. The PI through line overloads is defined as given below:

$$
P I=\sum_{i=1}^{n b} w_{i}\left(\frac{P_{l, \text { flow }}}{P_{l, \text { max }}}\right)^{2 n}
$$

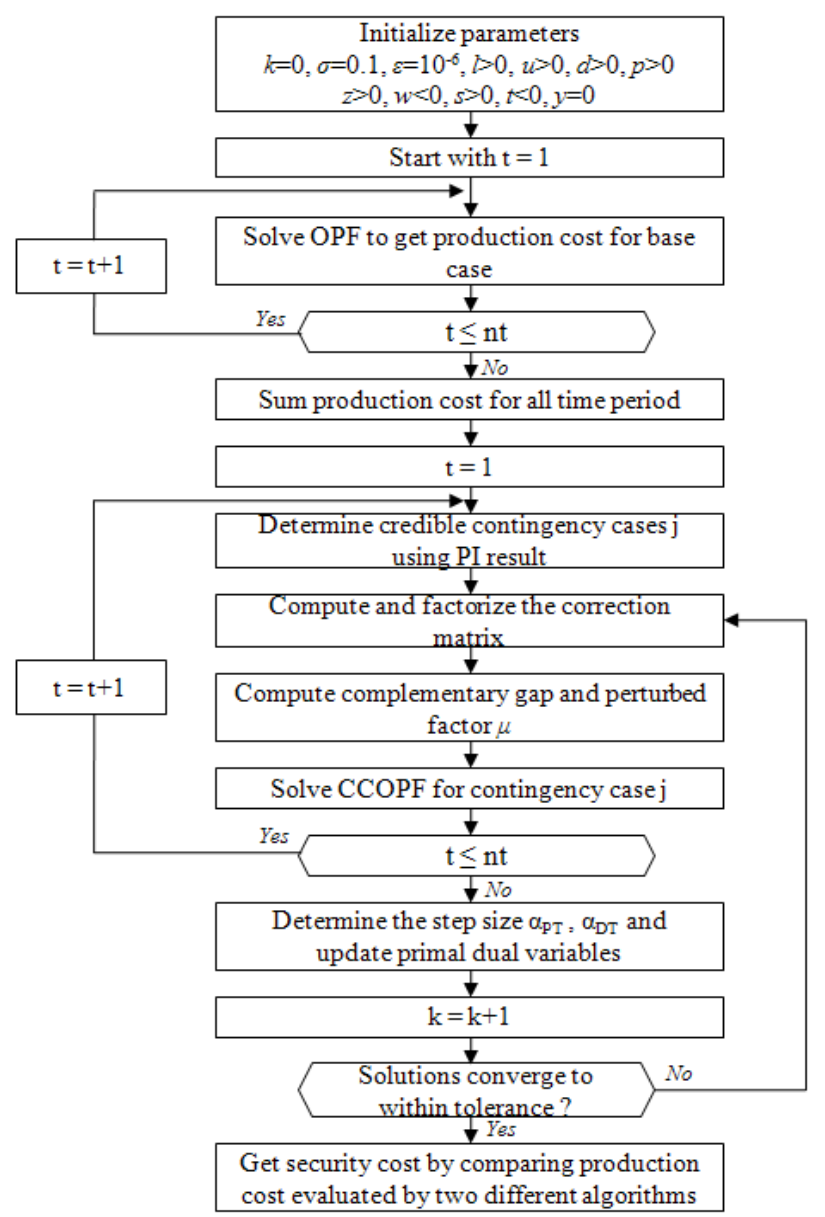

Fig. 2. The implementation of security cost analysis

where $\mathrm{P}_{l, \text { flow }}$ and $\mathrm{P}_{l, \text { max }}$ are the monitored line flow after an outage in line $l$ occurred and the maximum line flow for line $l$ respectively. The weighting coefficient $\mathrm{w}_{i}\left(0<\mathrm{w}_{i}<1\right)$ accommodates the importance of some power network devices. It is common to set the weighting coefficient to unity or to determine it from experience with the given network. Here, $n$ is a positive integer defining the exponent of the PI. For this study $n=1$. If the value of PI is large, it means the corresponding contingency is critical. The greater the value is, the more severe the contingency would be. Once PI has been measured, the results for PI are ordered from a large value to a small one. Thus, it provides a good measure of the severity of the line overloads for a given state of the power system. The algorithm of the proposed method is shown in Fig. 2. 


\section{Numerical Result}

The effectiveness of the CCOPF with a ramp limit for performing a security cost analysis was demonstrated in the IEEE-30 bus system, which consists of 6 generators and 41 lines, as shown in Fig. 3. The Appendix provides the characteristics of the generators and the load distribution over a 2-hour interval around the time of peak load in the test system. Since the economic dispatch problem is usually solved minute by minute, the security cost is evaluated every five minutes to determine the most economical scheduling considering the contingency constraint and other physical constraints. The results of CCOPF with the ramping constraint are compared with those of SCOPF over the same array of base case and line outages.

In the base case, the transmission line contingencies are not considered, and the total production cost is $\$ 14,895.80$. Using the performance index, the four most severe results for N-1 contingencies are illustrated in Table 1. In this work, only transmission line outages were considered.

In practice, power producers operate with distinct ramping limits through different operating conditions. Accordingly, the following security cases were considered:

Case I ( $n c=1)$ : line 28-27possibly on outage

Case II $(n c=2)$ : line 28-27 or 2-6 possibly on outage

Case III $(n c=3)$ : line $28-27$ or $2-6$ or $10-20$ possibly on outage

Case IV $(n c=4)$ : line $28-27$ or $2-6$ or $10-20$ or $10-17$ possibly on outage

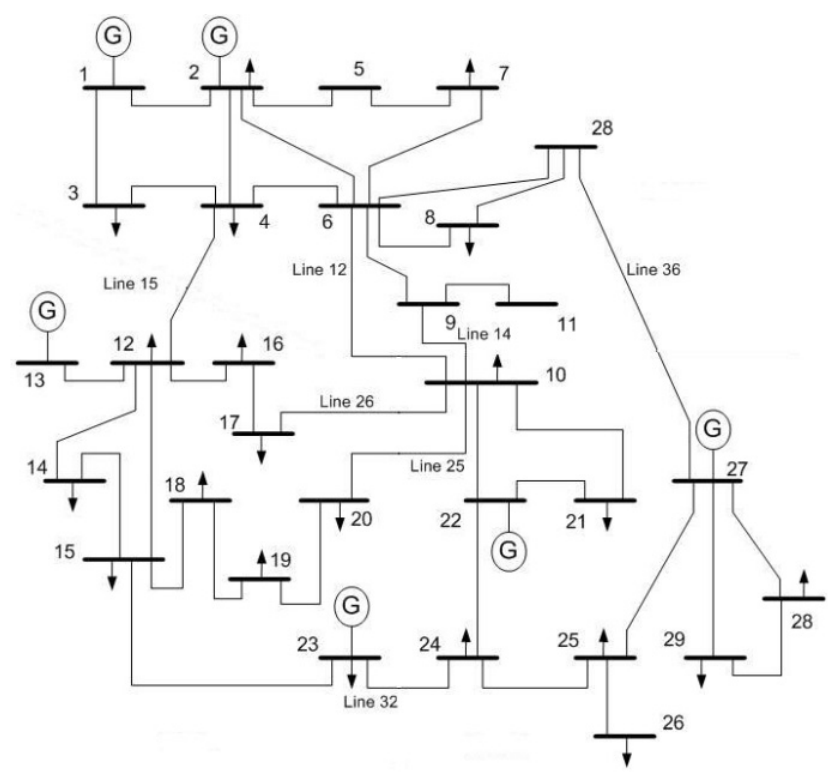

Fig. 3. IEEE-30 bus system

In Case IV, for example, we considered four single independent line outages. This case demands a contingency analysis for steady state transmission flow during each time period. The obtained CCOPF results were for the various contingency cases tabulated in Table 2. They include the power loss, production cost, and ramp rate viola- tions incurred by the power producers.

Clearly the production cost of electricity increases if we take into account transmission line contingencies and operational constraints. The security cost for each contingency case also increases with the number of possible contingencies. In this case study, the security cost had a value of approximately $1 \sim 2 \%$ of the production cost. That is why more expensive generators should cover the demand. Besides, the security cost of Case I is at most as high as a single contingency, because the outage of line $28-27$ is the most severe case. This result similarly associated with those of the performance index, which measure the severity of outage impacts. With an increase in the number of possible contingencies, the active power loss gradually becomes larger, and then pushes the security cost higher. Therefore, the security cost reflects not only active power loss but also the additional dispatch of an expensive generator.

Table 1. Contingency ranking

\begin{tabular}{ccc}
\hline Order of ranking & Outage line & PI \\
\hline 1 & $28-27$ & 0.058 \\
2 & $2-6$ & 0.056 \\
3 & $10-20$ & 0.045 \\
4 & $10-17$ & 0.019 \\
\hline
\end{tabular}

This implies that the feasible region of this optimization problem will become smaller with more possible contingencies. That is, this problem is limited by operational constraints, so its solution will be far from the optimal solution that can be obtained in unconstrained case.

As show in Table 2, the number of ramp rate violations seems to be not intimately linked with the number of possible outages. It could be related to the magnitude of the ramp rate and the cost coefficient of the generators. In fact, generator 4, which has the largest ramp rate, has never violated the ramp rate during any time period. However, generators 3 and 5, which have smaller ramp rates, often have their generation limited around times of peak load. Additional costs due to the ramping limit were about $3 \sim 5 \%$ of the security cost. Although the ramping effect is negligibly small with respect to the cost, it reflects the physical limitations of the generator. When the ramp rate constraint is considered, the proposed method can provide more realistic solutions.

Table 3 shows a comparison of security costs computed by the SCOPF and by the proposed method. It can be confirmed from the ratio of the security cost related to the production cost.

Table 2. Results for the contingency case

\begin{tabular}{ccccc}
\hline Case & $\begin{array}{c}\mathrm{P}_{\text {Loss }} \\
{[\mathrm{MW}]}\end{array}$ & $\begin{array}{c}\text { Number of } \\
\text { ramp rate } \\
\text { violations }\end{array}$ & PC [\$] & SC [\$] \\
\hline I & 78.9 & 11 & 15072.96 & 177.16 \\
II & 90.1 & 12 & 15116.832 & 221.03 \\
III & 95.4 & 11 & 15141.529 & 245.73 \\
IV & 98.7 & 13 & 15153.932 & 258.13 \\
\hline
\end{tabular}


From Table 3, since the SCOPF does not consider the ramp limit, the ramping cost is not charged. With the same security level, the security costs of SCOPF are larger than those of the proposed method. This result shows that the proposed method can help market participants become more competitive while maintaining the same security level as with SCOPF. Furthermore, power suppliers can determine their generation level within reasonable security levels and physical limits. Therefore, this approach is very attractive because the correct security cost information allows one to make more profitable decisions than with the conventional method for the trade-off between security and economics in electricity markets.

\section{Conclusions}

We have developed a model of security costs based on the ramping process. With this proposed approach, if we know the operating point at time $t-1$, the ramping constraint can be involved as an inequality. The feasibility of a CCOPF problem formulated by PDIPM was demonstrated with the IEEE-30 bus system. The main contribution of this paper is to show that using ramp rate constraints in the CCOPF algorithm will normally result in better economic outcomes (for consumers and producers) than the preventive method. Since the proposed method satisfies physical limits including the ramp limit and the power output limit, furthermore, it provides a more realistic and efficient optimal solution to ensure the operational feasibility of transactions. Therefore, the optimal solution of the proposed approach can help power suppliers make more profitable decisions with a reasonable security level and satisfy physical constraints at the same time. The ramping affect was negligible in comparison with the production cost, but this should be considered in a multi-period dispatch problem.

\section{Acknowledgements}

This work was supported by the Electrical Industry Research Center (EIRC) of the Ministry of Knowledge Economy (MKE) through the Advanced Power Network Reliability Research Center (homepage-http:// aprrc.snu.ac.kr)

\section{APPENDIX}

\section{A. GENERATOR DATA}

\begin{tabular}{cccccccc}
\hline \multicolumn{7}{c}{ Unit Cos Coefficients } \\
Unit & Bus & $\begin{array}{c}\mathrm{A} \\
\text { ABtu })\end{array}$ & $\begin{array}{c}\mathrm{B} \\
(\mathrm{MBtu} / \\
\mathrm{MWh})\end{array}$ & $\begin{array}{c}\mathrm{C} \\
(\mathrm{MBtu} /\end{array}$ & $\begin{array}{c}\mathrm{P}_{\max } \\
{[\mathrm{MW}]}\end{array}$ & $\begin{array}{c}\mathrm{P}_{\min } \\
{[\mathrm{MW}]}\end{array}$ & $\begin{array}{c}\text { Ramp rate } \\
{[\mathrm{MW} / \mathrm{min}]}\end{array}$ \\
\hline $\mathrm{G} 1$ & 1 & 0.02 & 2 & 0 & 80 & 0 & 1.5 \\
$\mathrm{G} 2$ & 2 & 0.0175 & 1.75 & 0 & 80 & 0 & 2 \\
$\mathrm{G} 3$ & 13 & 0.025 & 3 & 0 & 40 & 0 & 1 \\
$\mathrm{G} 4$ & 22 & 0.0625 & 1 & 0 & 50 & 0 & 3 \\
$\mathrm{G} 5$ & 23 & 0.025 & 3 & 0 & 30 & 0 & 1 \\
$\mathrm{G} 6$ & 27 & 0.00834 & 3.25 & 0 & 55 & 0 & 2 \\
\hline
\end{tabular}

\section{B. LOAD DATA}

\begin{tabular}{cccccccc}
\hline $\begin{array}{c}\text { Time } \\
\text { period }\end{array}$ & $\begin{array}{c}\text { Total } \\
\text { load }\end{array}$ & $\begin{array}{c}\text { Time } \\
\text { period }\end{array}$ & $\begin{array}{c}\text { Total } \\
\text { load }\end{array}$ & $\begin{array}{c}\text { Time } \\
\text { period }\end{array}$ & $\begin{array}{c}\text { Total } \\
\text { load }\end{array}$ & $\begin{array}{c}\text { Time } \\
\text { period }\end{array}$ & $\begin{array}{c}\text { Total } \\
\text { load }\end{array}$ \\
\hline 5 & 149.9 & 35 & 188.7 & 65 & 238.3 & 95 & 234.2 \\
10 & 147.4 & 40 & 169.8 & 70 & 264.8 & 100 & 236.5 \\
15 & 117.1 & 45 & 161.3 & 75 & 286.7 & 105 & 231.8 \\
20 & 128.8 & 50 & 145.2 & 80 & 292.7 & 110 & 208.6 \\
25 & 167.4 & 55 & 152.5 & 85 & 289.8 & 115 & 185.6 \\
30 & 192.5 & $1 \mathrm{hr}$ & 190.7 & 90 & 260.8 & $2 \mathrm{hr}$ & 188.3 \\
\hline
\end{tabular}

\section{References}

[1] Song, Y. H and Wang, X. F, Operation of Marketoriented Power Systems, Springer. 2003.

[2] Tamimi, B. and VaezZadeh, S., An Optimal Pricing Scheme in Electricity Markets Considering Voltage Security Cost, IEEE Transactions on Power Systems, Vol. 23, No. 2, May 2008.

[3] Shariati, H., Askarian, A. H, Javidi, M. H. and Razavi, F, Transmission expansion planning considering security cost under market environment, Electric Utility Deregulation and Restructuring and Power Technologies, April 2008.

[4] Hong, C., Canizares, C. A and Singh. A., Web-based security cost analysis in electricity markets, IEEE Transactions on Power Systems, Vol. 20, No. 2, May 2005.

[5] Wang, C. and Shahidehpour, S. M., Effects of Ramprate limits on Unit Commitment and Economic Dispatch, IEEE Transactions on Power Systems, Vol. 8, No. 3, Aug. 1993.

[6] Alva, J. S., Tseng, C. L., Li, C. A. and Raymond B. Johnson, Short-Term Resource Scheduling with Ramp Constraints, IEEE Transactions on Power Systems, Vol. 12, No. 1, Feb. 1997.

[7] Han, X. H, Gooi, H. B. and Venkatesh, B., Dispatch Problems Due to Ramp Rate Constraints: Bottleneck Analysis and Solutions, Electric Power Components and Systems, Vol. 31, No. 10, Oct. 2003.

[8] Kim, M. K., Hur, D., and Park, J. K., Determination of available transfer capability using multi-objective contingency constrained optimal power flow with post-contingency corrective rescheduling, Electrical Engineering, April, 2008.

[9] Anitha, M., Subramanian, S., and Gnanadass, R., Optimal production cost of the power producers with linear ramp model using FDR PSO algorithm, European Transactions on Electrical Power, Oct. 2008.

[10] Hua, W., Sasaki, H., and Kubokawa, J., An Interior Point Nonlinear Programming for Optimal Power Flow Problems with A Novel Data Structure, IEEE Transactions on Power Systems, Vol. 13, No. 3, Aug. 1998.

[11] Yamin, H. Y., Dynamic Optimal Power Flow Using Interior Point Method and Benders Decomposition Considering Active and Reactive Constraints, Electric Power Components and Systems, Vol. 34, No. 12, Dec. 2006. 
[12] Lin, W. M, and Chen, S. J., Bid-based dynamic economic dispatch with an efficient interior point algorithm, Electrical Power and Energy Systems, Vol. 24, 2002.

[13] Stott, B., Alsac, O., and Monticelli, A.J., Security analysis and optimization, Proceedings of the IEEE, Vol. 75, No. 12, Dec. 1987.

Jae-kum Lyu received his B.S. degree in electrical engineering from Shinshu University, Nagano, Japan in 2006. Currently, he is working on his Unified Master's and Doctor's Course in the Department of Electrical Engineering at Seoul National University. His research field of interest includes power system security and system margins.

Mun-Kyeom Kim received B.S. degree in Electrical Engineering from Korea University, Seoul, Korea in 2004 and his M.S. degree in Electrical Engineering from Seoul National University, Seoul, Korea in 2006. He is currently working on his Ph.D. degree in the School of Electrical Engineering at Seoul National University. His research interests are in the areas of power system restructuring, power system operation, and risk management in the deregulated electricity markets.
Jong-Keun Park received his B.S. degree in electrical engineering from Seoul National University, Korea in 1973 and his M.S. and Ph.D. degrees in electrical engineering from The University of Tokyo, Japan, in 1979 and 1982, respectively. From 1983, he has worked as an assistant professor, an associate professor and a professor in the School of Electrical Engineering, Seoul National University, Korea. His research areas are power system economics, energy policy and power system analysis. He is a senior member of the Institute of Electrical and Electronics Engineers (IEEE) and a fellow of the Institute of Electrical Engineers (IEE). He is also Vice President of the Korean Institute of Electrical Engineers (KIEE), a member of the National Academy of Engineering of Korea and the Korean representative of the study committee SC5 "Electricity Markets and Regulation" in CIGRE. 